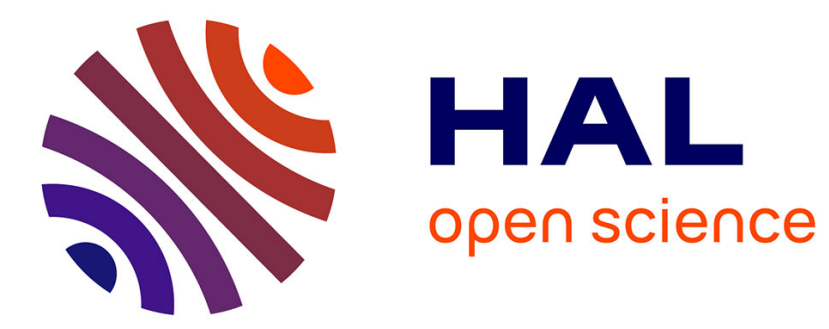

\title{
Fragment d'inscription d'Allonnes (Sarthe)
}

Albert Grenier

\section{To cite this version:}

Albert Grenier. Fragment d'inscription d'Allonnes (Sarthe). Gallia - Fouilles et monuments archéologiques en France métropolitaine, 1957, 15 (1), pp.145-146. 10.3406/galia.1957.1494 . hal01923727

\section{HAL Id: hal-01923727 \\ https://hal.science/hal-01923727}

Submitted on 3 Mar 2020

HAL is a multi-disciplinary open access archive for the deposit and dissemination of scientific research documents, whether they are published or not. The documents may come from teaching and research institutions in France or abroad, or from public or private research centers.
L'archive ouverte pluridisciplinaire HAL, est destinée au dépôt et à la diffusion de documents scientifiques de niveau recherche, publiés ou non, émanant des établissements d'enseignement et de recherche français ou étrangers, des laboratoires publics ou privés.

\section{(이) $\$$}

Distributed under a Creative Commons Attribution - NonCommercial - NoDerivatives| 4.0 


\section{DOCUMENTS COMMENTÉS}

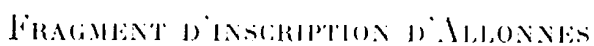
(somTur).

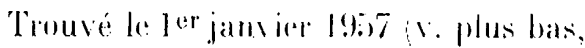
P. Cordonnier-1)elrie, Informalions. p. 2ors.

le nom du personnage manque ainsi que de déloul de sa rarriere lig. I.

1 |cenlurion leg(ionis) I kimlen!sls X gieminate

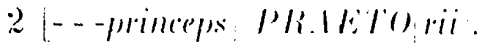

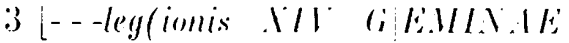

II arliae Victricis:

4.--praeposilus vexi]LL.1TLON[ibus]

b L-- -leg(ionis III G]ALI.ICALe:

6 [leg. undecima $E$ CLLandiae

7 L- - - leg(ionis - - ? | HE

$8[---]$ C ou $0[--]$.

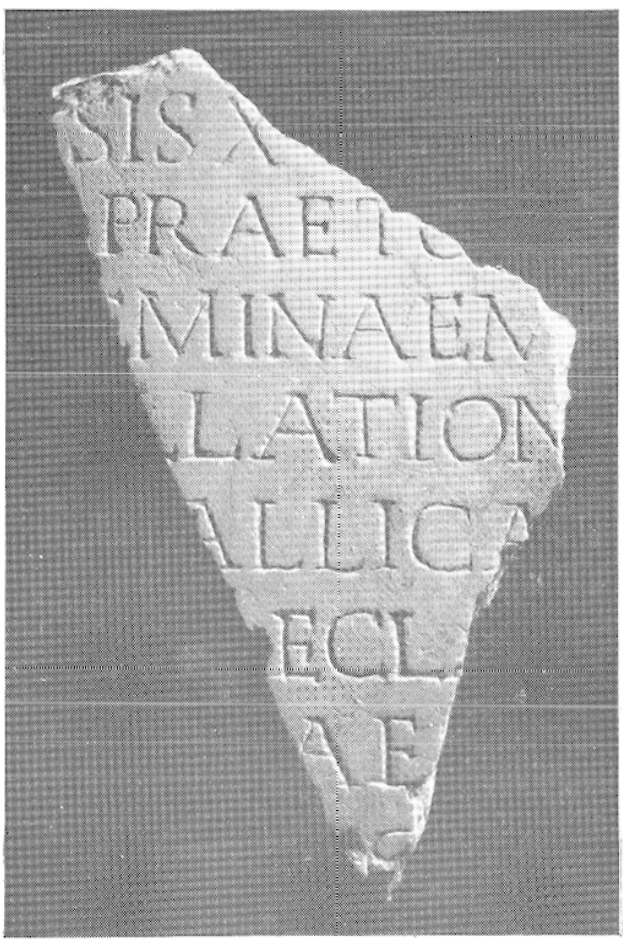

1. 1. Le nom de la légion est loujours $X$ Fretensis. Le $X$ qui suit el qui ne paraìt pas suivi d'unités ne peut-être que la X" Comina, la seule a porter le numéro $X$. La $X$ e Frelensis, longlemps ren syrie a pris parl sous Marc-Aurele a la guerre des Mareomans. La Xo Ciemina, aprés avoir lail parlice de l'armée de Germanie Inférieure, a lenu au ${ }^{\circ}$ siede garnison is lindobonde (Vienne) el à Carmunlum en Pannonie, sur le l)anube.

1. 2. Le princeps praelorii est un centurion généralement primipile chargé de l'administration du prétoire et des magasins d'armes et aulres qui s'y trouvent concentrés.

1. 3. Ouatre légions portent ce litre de Gemina. Le $M$ qui suil indique la XIVe qui, à parlir de Trajan a porté le

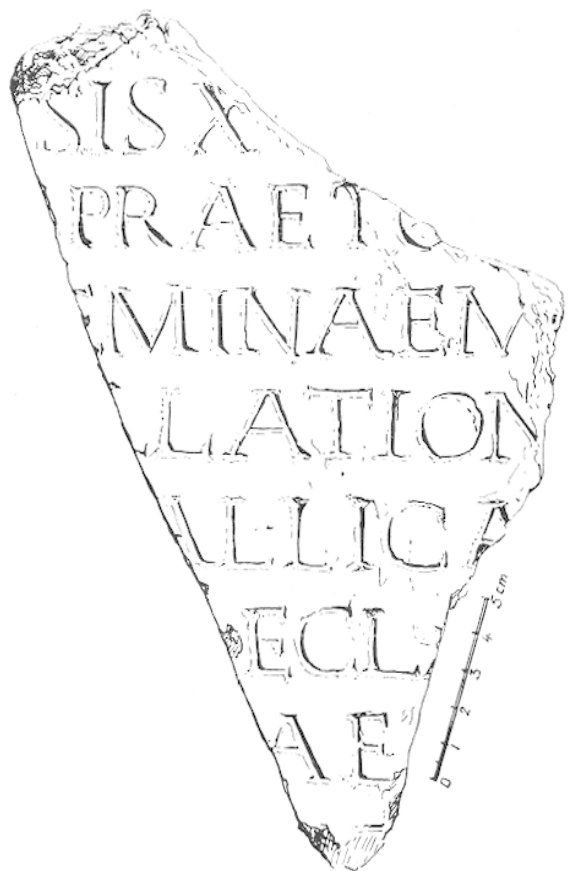

fïg. 1. Allomes. Fragment d'inscription sur marbre. 
Litre de Martia el même Marlia Victrix. et a tenu longtemps garnison en Pannonie, avec la Xe Gemina (l. 1).

1. 1. Des centurions primipiles peuvent être chargés du commandement de délachements ou, comme ici, de groupes de délachements; cl. 1)essau, Inscr. seleclae, $\mathrm{n}^{0 ;} 1387$ et 2726 .

1. 5. Parmi les dillérentes légions qui ont porté lemporairement, le litre de Gallica, la II [e dont, c'est le tilre régulier peul se:ale entrer irei en ligne de comple.

1. 6. Trois légions ont porté le libre de Clandia, je crois pouvoir préférer la $X I 0$ qui a tenu garnison a Cirnumlum sur le: I)ariube.

1. 7 et 8 restent indélerminables.

I.e texte, d'apres l'écrilure est de la lin du $11^{\circ}$ ou du début du $\mathrm{II}^{\mathrm{e}} \mathrm{s}$. ... l.e renlurion a dì servir dans l'armée du Danube. D'apres les délachements groupes sous son commandement, il pourrail, s'agir des rébuls du régne de Septimesevere, peul-ille de sa luble contre Jidius Julianus.

Mllert GiRsitils.

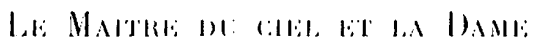 BI: VII:?}

La leclue de: l'ouvrage que M. l'.-M. Duval vient de consacrer aux dieus de la ciaule minvile a publier une nole, depuis longlemps preparée ${ }^{2}$ sur un relief conservé au Musce d'Hyeres.

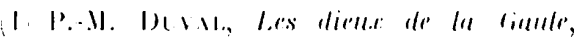
l'. I. II, I!I:7.

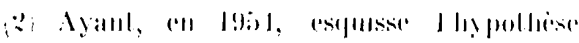
devant la Socirte Archiologigue de Bordeaux, je posais a propos du meme documenl, devant

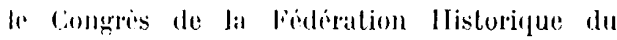
sud-(Onest tenu a sainl-sever en 1956, la queslion du caractire ligure ou cellique.
M. R. Lanlier a présenté ainsi cette piece $^{3}$ :

"Bas-relief mulile lronvé à Ilyères. all quarlier d'Almanarre, sur l'emplacement d'olbia (Var). A" Musée d'Myères. Pierre commune. Hauleur; $0 \mathrm{~m} .36$; Largeur, $0 \mathrm{~m}$. 30); Épaisseur, () $\mathrm{m}$. I:3. Pholographie el renseignemenls commmuniqués par M. F. Benoil. A droile, Allante (?) deboul, lo lorse mu, un calegon court el plissé serré an.e hamches, le genou droil plie à angle alroil. La grosse face lunaive esl encadrée par les frisoms d'une barbe grossieremenl Irailée an Iripan. less avanl-bras el les jambes mannulenl. I gauche une femme deloml. Ir lorse inl ; la parlie inferteure du comps. brisese anar genoux, est embeloppee dans ane draperie donl fextrémile supérienure s'emroule en bermorelel andoun des hanches. Jans les

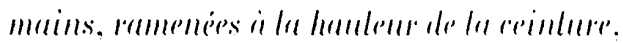

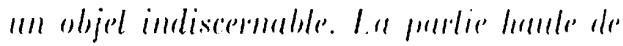
la chereluere a disparta $n^{4}$.

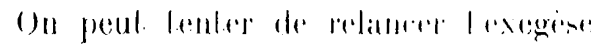

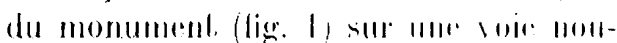
valle.

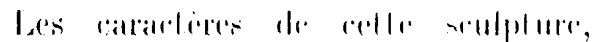
schemalisme tres dementante. mancer céphalie, exophlalmire trailement de ba barbe dans la tradilion de la loreuliguar,

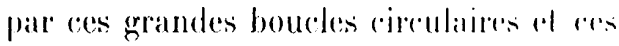
coups de lrépan, dénoneratenl asser. bien une aruvere de haule epoque galloromaine, on loul cas d'and delespril indiginessi.

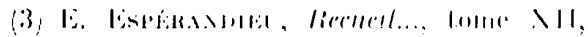

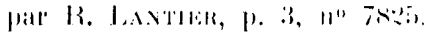

(1) Ajoutoms am imbleations dommes pall

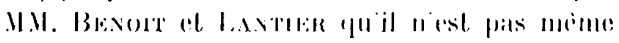

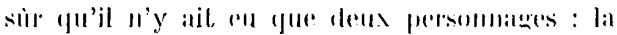
pierre est de lous coles rolionement mulilere.

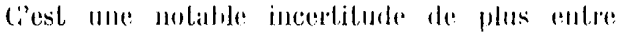
loutes celles qui nous interdiront quelquar antclusion ferme.

(5) C.1. en dernier lieu .J.-.. IIsTr, R. li. .1., I.1X, 1957, p. 8.3 el suiv. 